\title{
ESTABILIDAD DE LOS AGREGADOS DE UN SUELO QUEMADO A DIFERENTES INTENSIDADES DE FUEGO DIECIOCHO AÑOS DESPUÉS DE UN INCENDIO FORESTAL
}

\author{
A. VELASCO, X. ÚBEDA* \\ GRAM (Grup de Recerca Ambiental Mediterrània) \\ Departament de Geografia Física i AGR \\ Universitat de Barcelona. Montalegre 6, 08001 Barcelona, Spain.
}

\begin{abstract}
RESUMEN. En 2012, dieciocho años después de un incendio forestal que tuvo lugar en 1994 y quemó una plantación de Pinus pinaster, se analiza la estabilidad de los agregados del suelo comparándolos con los agregados de una zona control sin quemar. El área quemada, justo después del incendio, se dividió en tres zonas, baja, media y alta intensidad de fuego, dependiendo de la intensidad a la que se quemaron cada una de las tres áreas. Los resultados de este muestreo en 2012 se comparan con los análisis de los agregados del suelo efectuados en 1994 y en 1995. Los resultados muestran que dieciocho años después del incendio los agregados son menos estables en las zonas quemadas basados en dos métodos de análisis y que las diferencias entre las zonas quemadas no son significativas. El hecho que el suelo de la zona control tenga más materia orgánica y más concentración de elementos químicos, como el calcio, magnesio y hierro, parece ser el factor determinante de una mayor estabilidad de los agregados más superficiales en la zona control.
\end{abstract}

Water-stable aggregates in a burned soil at different intensities eighteen years after a forest fire

ABSTRACT. In 2012, eighteen years after a forest fire that took place in 1994 and burned a Pinus pinaster plantation, the soil aggregate stability is analysed and compared with aggregates of an unburned control area. The burned area was divided into three zones just after the fire, low, medium and high intensity fire, depending on the intensity at which burned each of the three zones. The results of the sampling in 2012, were compared to the analysis of the soil aggregates in 1994 and 1995. The results show that after eighteen years the aggregates of the burned zones are less stable based on two analytical methods, and the differences between burned areas are not significant. The fact that the soil of the control zone has more organic matter and concentration of chemical elements like, calcium, magnesium and iron, seems to be the determining factor of greater stability of surface aggregates in the control zone. 
Palabras clave: efectos del fuego a corto y largo término, métodos TDI y CND, macizo de Cadiretes, Girona.

Key words: short and long term fire effects, TDI and CND methods, Cadiretes massif, Girona.

Enviado el 15 de noviembre de 2013

Aceptado el 20 de febrero de 2014

*Correspondencia: GRAM (Grup de Recerca Ambiental Mediterrània) Departament de Geografia Física i AGR, Universitat de Barcelona, Montalegre 6, 08001 Barcelona, Spain. E-mail: xubeda@ub.edu

\section{Introducción}

Los agregados del suelo son agrupaciones de partículas tanto minerales como orgánicas. Los coloides, cargados eléctricamente, son los núcleos de estos agregados y debido a diferentes procesos de floculación dan lugar, primeramente, a microagregados $(<0,25 \mathrm{~mm})$ que pueden evolucionar y crecer en tamaño, llegando a definir la estructura del suelo. En el proceso de agregación toman parte tanto factores abióticos (mineralogía de las arcillas, humedad, etc.) como biológicos (animales y vegetales). La complejidad del estudio de la estructura del suelo se debe precisamente al elevado número de factores que intervienen (Mataix-Solera et al., 2010). Para que un suelo pueda cumplir satisfactoriamente las funciones relacionadas con procesos físicos, químicos y biológicos, la formación de agregados estables es de vital importancia (Mataix-Solera y Guerrero, 2007).

La estabilidad estructural de agregados se define como: "la resistencia de los agregados del suelo a desintegrarse por la acción de fuerzas externas (por ejemplo el impacto de gotas de lluvia o la humedad)". Por ello, esta medida se ha utilizado como un indicador de la estabilidad física del suelo (Imeson, 1984). Son muchas las propiedades que se pueden ver afectadas tras un incendio forestal y muchas de estas propiedades están ligadas a la estabilidad estructural, como la materia orgánica, ya que su cantidad y calidad controlan la agregación de partículas (Oades, 1993).

Hay otros componentes importantes para la creación y estabilidad de los agregados como el contenido en arcilla y la mineralogía de las fracciones (Singer, 1994) o los microorganismos que influyen en dicha formación (Harris et al., 1964; Insam y Domsch, 1988).

Los incendios, si son muy intensos, pueden llegar a desestructurar los agregados (Hillel, 1998). Debido a la destrucción de agregados puede generarse un sellado superficial del suelo y provocar grandes cambios en su superficie (McIntyre, 1958; Certini, 2005). Este hecho puede provocar un descenso en la infiltración del agua y, por otro lado, un incremento de la erosión (Hoogmoed y Stroosnijder, 1984). Incluso algunos centímetros de suelo pueden ser removidos debido a dicha erosión, con lo que horizontes habitualmente profundos quedan expuestos a la atmósfera (Mataix-Solera y Cerdà, 2009).

Los cambios que puede sufrir la estabilidad estructural después de un incendio forestal son difíciles de evaluar. Esta depende de muchos factores, entre ellos las propiedades del sue- 
lo o la intensidad del incendio. Este hecho ha dado lugar a discrepancias y, sobre todo, a una gran diversidad de resultados. Por ello se hace necesario estudiar numerosos factores implicados en el proceso para poder llevar a cabo una interpretación correcta de dichos resultados. Dependiendo de la severidad del incendio y el tipo de suelo, los cambios serán más o menos evidentes. Generalmente se observa que el fuego produce una fragmentación de agregados y una pérdida de estabilidad debido a la ruptura de los cementos orgánicos a temperaturas elevadas (Badía y Martí, 2003). Incluso se ha podido reportar que los agregados supervivientes pueden mostrar una estabilidad superior a los de antes del incendio debido a la formación de óxidos de cementación (Giovannini y Lucchesi, 1997; Ketterings et al., 2000). En algunos casos, sin embargo, no se han observado diferencias (por ejemplo en incendios que se propagan a través de las copas de la vegetación arbórea, que pueden producir un aumento de la materia orgánica edáfica) y en otros los resultados han mostrado una agregación mayor en los suelos quemados a intensidades bajas y medias debido a la formación de una capa hidrofóbica en la superficie externa de los agregados (Mataix-Solera y Doerr, 2004).

Debido a la elevada complejidad de esta propiedad edáfica, no existe un acuerdo general sobre el método analítico más apropiado para su determinación (Bryan, 2000). Por ello encontramos un gran número de metodologías y técnicas diferentes. Algunas están enfocadas hacia la distribución de los agregados por tamaños y otras hacia la estabilidad de los mismos. En el presente artículo resumimos en esta introducción los métodos divididos en cinco grupos, todos ellos recogidos en el libro "Actualización en métodos y técnicas para el estudio de los suelos afectados por incendios forestales", editado por Artemi Cerdà y Antonio Jordán (2010).

El primer grupo utiliza la técnica de la tamización en seco (Kemper y Rosenau, 1986). En este método se calcula el \% de agregados de cada una de las fracciones así como el diámetro medio ponderado según la expresión propuesta por los autores citados.

El segundo grupo se basa en el método de la tamización en húmedo. El más utilizado es el derivado del propuesto por Yoder (1936). Consiste en medir la cantidad de agregados que permanecen intactos después de que la muestra de suelo se somete a la acción de fuerzas mecánicas por tamización en húmedo. Existen muchas variantes de este método. Se pueden utilizar uno o varios tamices. También varía la cantidad de muestra utilizada, la frecuencia de las oscilaciones o el tiempo de duración del tamizado, entre otras. Kemper y Koch (1966) propusieron un test de estabilidad estándar para medir los macroagregados estables al agua. Este fue mejorado por Kemper y Rosenau (1986) posteriormente.

El tercer grupo muestra los métodos basados en los impactos repetidos de gotas de agua sobre agregados individuales. Low (1954) determinó la estabilidad de los agregados basándose en su respuesta frente al impacto de gotas de agua. Estos métodos permiten cuantificar con mayor exactitud la energía aplicada y el modo de actuación de la misma. Las simulaciones de lluvia se emplean en la medida de la estabilidad de los agregados al agua, aunque inicialmente se habían pensado para estudiar la erosión hídrica. La desventaja principal de este método es la falta de estandarización, lo que dificulta la comparación de resultados. Existen diversos métodos, siendo el CND, que cuenta el número de impactos de gota (Low, 1954) y el TDI, que mide la disgregación ante diez impactos de gota (Imeson y Vis, 1984), los más importantes. 
El cuarto grupo lo forman aquellos métodos que emplean simulaciones de lluvia. Los principales autores de estos métodos son Benito et al. (1986). Métodos posteriores han estado, con frecuencia, basados en el primero. Este consiste en colocar $50 \mathrm{~g}$ de suelo tamizado a $2 \mathrm{~mm}$ en tamices de $15 \mathrm{~cm}$ de diámetro y $0.25 \mathrm{~mm}$ de luz y posteriormente, someterlos a la lluvia simulada durante 30 minutos. Tras este proceso se debe secar el material en una estufa a $105^{\circ} \mathrm{C}$ hasta alcanzar un peso estable. El test permite contabilizar la masa de agregados que permanece en el tamiz. Los resultados se pueden expresar como pérdida de suelo en $\mathrm{g} \mathrm{m}^{-2} \mathrm{~min}^{-1} \mathrm{o}$ como $\%$ de agregados estables.

En el último grupo están aquellos métodos de estabilidad de agregados que emplean ultrasonidos. Estos consisten en sumergir los agregados en agua y aplicarles una dosis de energía controlada por medio de una fuente de ultrasonidos, cuantificando los agregados que resisten al tratamiento. Entre los autores más destacados tenemos a Edwards y Bremner (1967) y Holz et al. (2000), que definieron diferentes metodologías que utilizaban ultrasonidos. Los resultados se expresan como el porcentaje de agregados estables tras el tratamiento con ultrasonidos, con respecto al total de agregados presentes en la muestra inicial.

Hay otros métodos interesantes de ser citados como el Método de las rotaciones. El método de las rotaciones informa sobre la estabilidad de los agregados en condiciones semejantes a las que se produce su transporte fluviotorrencial (Salvador Sanchís et al., 2008). La metodología utilizada se basa en el humedecimiento por capilaridad de 30 gramos de muestra de suelo (capacidad de campo), previamente tamizada entre 0,5 y $8 \mathrm{~mm}$. Una vez seleccionado el material, mediante el tamizado, se deposita en unas bandejas y se seleccionan $30 \mathrm{~g}$ de muestra. Posteriormente, se introduce la muestra en contenedores cerrados herméticamente (de $10 \mathrm{~cm}$ de altura y $10 \mathrm{~cm}$ de diámetro de la base) y se añade un total de $200 \mathrm{~cm}^{3}$ de agua desionizada. Una vez cerrados, los contenedores se acoplan a un rotor. La muestra se agita durante un tiempo conocido (calculado previamente), dependiendo del número de ciclos aplicados (Salvador Sanchís et al., 2008). Después del número de rotaciones previamente preestablecido para el estudio (por ejemplo: 20, 60, 180,540 y 1600 rotaciones) el contenido del recipiente se transfiere a una torre de tamices. Normalmente son utilizados los siguientes tamices: 2000, 1000, 500, 250, 125 y 63 micras. A continuación, se lava la muestra con agua desionizada para facilitar el paso de los agregados a través del tamiz. El material obtenido se deja secar en la estufa a $105^{\circ} \mathrm{C}$. Una vez seco el material, se pesan los recipientes. Los datos relativos a la estabilidad estructural de los agregados se pueden describir en términos de dimensión de los agregados (diámetro D50 correspondiente a la mediana de la distribución de los agregados). Para el análisis de los datos adquiridos a partir de este procedimiento se puede realizar un estudio de la distribución del tamaño de los agregados residuales (en las 7 categorías obtenidas a partir de los 6 tamices utilizados) y el cálculo por interpolación del diámetro mediano de los agregados (D50), que se toma como el parámetro guía para determinar su estabilidad (Salvador Sanchís et al., 2008).

Hay poca bibliografía sobre efectos de los incendios forestales a largo término, así que creemos que este artículo puede ser una contribución más a conocer el estado del suelo en zonas quemadas y en las cuales no se ha realizado ningún tipo de gestión postincendio de aprovechamiento de la madera ni de revegetación o reforestación. 
El objetivo de este artículo es comprobar cuál es el estado de la estabilidad estructural en el suelo de un área quemada hace 18 años, considerado como un largo periodo de tiempo desde el impacto fuego. Además, se parte de la división de esta parte quemada en 3 zonas diferentes en intensidad de quemado, para comprobar si los efectos han sido homogéneos en toda el área quemada. Los resultados se comparan con la estabilidad estructural de un suelo control.

\section{2. Área de estudio}

El estudio que se presenta en este artículo está basado en un trabajo realizado sobre un incendio en el macizo de Cadiretes (Úbeda, 1998), localizado en el término municipal de Llagostera, Girona (Fig. 1). El incendio de "Llagostera" comenzó el mediodía del día 5 de julio de 1994 y fue extinguido en seis horas, quemando un total de 55 hectáreas de bosque, atribuyendo la causa del incendio a un hecho intencionado.

En la Fig. 1 se muestra también dónde tuvo lugar el incendio, dentro de la propiedad de Can Noguera, en las montañas de Llobatera. El punto más alto que resultó incendiado se sitúa a 350 metros sobre el nivel del mar. La carretera que une Llagostera con Tossa de Mar delimita el incendio por la parte más baja de la vertiente quemada. Dos torrentes que solo llevan agua en episodios muy concretos de lluvia hacen de desagüe de la escorrentía y la erosión del área quemada.

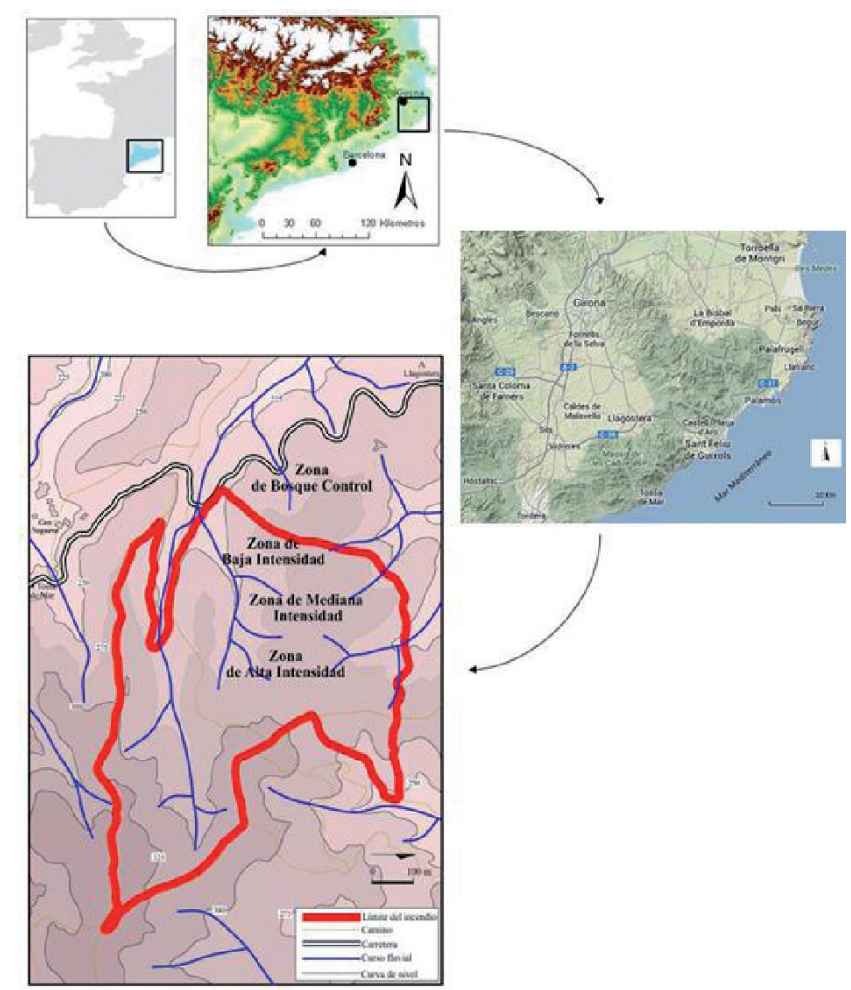

Figura 1. Localización del área de estudio y zonas de muestreo. 
En la finca de Can Noguera no hay cortafuegos, pero sí numerosos caminos en toda la propiedad. Los caminos son transitables con turismo todo terreno, habiendo limitación en alguna pista forestal que tiene pendientes de hasta el $16 \%$, siendo estos los sectores que presentan mayores problemas de erosión. Los caminos forestales conectan con la carretera que va a Llagostera.

El macizo de Cadiretes está dentro de la zona del alcornoque (Quercetum ilicis galloprovinciale suberetosum). Es un bosque poco denso de alcornoques (Quercus suber), pero muy densamente poblado de sotobosque; lo que lo hace difícilmente transitable. El sustrato silíceo y de carácter ácido favorece el desarrollo del alcornocal juntamente con el sotobosque típico de especies silicícolas. Los alcornoques también están presentes en el área quemada, notándose mucho más su presencia una vez el fuego eliminó completamente al Pinus pinaster en algunos lugares. El bosque que se quemó en julio de 1994 era una plantación de Pinus pinaster, con ejemplares muy bien alineados sobre terrazas hechas exclusivamente para esta finalidad. Las copas de estos árboles se tocaban unas a otras desde la parte más baja hasta la parte superior de la vertiente.

El sotobosque de este pinar y alcornocal está compuesto por arbustos como el Arbutus unedo, Erica arborea, Rubia peregrina, Smilax aspera, Lonicera implexa y Ruscus aculeatus. Además hay un importante estrato herbáceo predominantemente compuesto por Brachypodium.

El sustrato del área quemada está constituido por rocas metamórficas del paleozoico, que no es la litología representativa del macizo de Cadiretes, ya que es el granito el que ocupa su mayor parte. El suelo se ha clasificado según la Soil Taxonomy como un Lithic Xerochrept.

\section{Metodología}

\subsection{Determinación de la intensidad de fuego}

Inmediatamente después del incendio en 1994, y una vez los trabajos de extinción del incendio concluyeron, las diferentes intensidades de fuego fueron determinadas en el campo. Se distinguieron tres zonas mediante la observación del estado de los árboles y de las cantidades de ramas y hojas que permanecían en los árboles, así como la cantidad de hojarasca que había depositada en la superficie del suelo (Moreno y Oechel, 1989). Después del incendio, la vegetación en las diferentes zonas tenía las siguientes características (Fig. 1):

- Baja o de baja intensidad de fuego: Los árboles (Pinus y Quercus) mantenían algunas hojas (aunque no fueran totalmente de color verde), y un gran número de ramas, incluso pequeñas. Mucha hojarasca cubrió el suelo justamente después del incendio y no llegó a quemarse. Los Quercus suber sobrevivieron y también algún Pinus pinaster. Esta zona está localizada alrededor de los torrentes y en la parte más baja de la vertiente. Debajo de la hojarasca depositada se podían observar la presencia de cenizas de color muy negro, que aún perduraban en muchos lugares dos años y medio después del incendio.

- Media o de mediana intensidad de fuego: Los árboles no tenían ninguna hoja, pero conservaban un número importante de ramas. No había mucha hojarasca en el suelo. 
Esta zona se sitúa alrededor de la zona de baja intensidad y en la parte superior de la vertiente. Las cenizas de color negro aún se conservaban en varios puntos treinta meses después del incendio.

- Alta o muy alta intensidad de fuego: Los árboles habían perdido todas las hojas y ramas. El Arbutus unedo había desaparecido, cosa que no pasaba en las otras zonas. Justo después del incendio, la superficie del suelo estaba cubierta por ceniza de color gris y blanco que desapareció rápidamente después de las primeras lluvias, hecho por el cual la superficie quedó totalmente desprotegida.

En la actualidad, el área quemada presenta una densa vegetación. Hay un importante rebrote de pinos y un sotobosque muy densamente poblado por especies arbustivas, Erica arborea, Arbutus unedo, Cistus salvifolius, Cistus monspeliensis, Calicotome spinosa y Brachypodium retusum entre otras. Se puede decir que la estructura actual de este joven bosque y su continuidad horizontal y vertical lo hace un área de alto riesgo de gran incendio forestal.

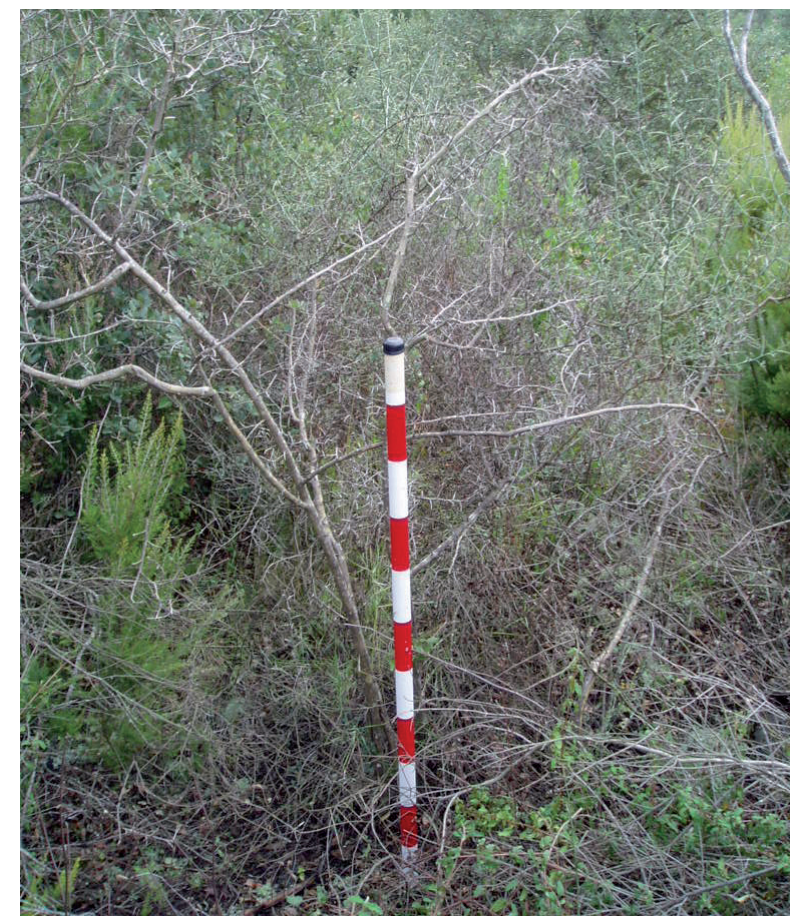

Figura 2. Fotografía del estado actual del bosque quemado en 1994.

\subsection{Muestreo de suelo y métodos de análisis}

En diciembre de 2012 (dieciocho años aproximadamente después del fuego) se realizó una jornada de campo para recoger muestras de suelo de las cuatro zonas de estudio (Baja, Media, Alta intensidad y Control). 
Las muestras en cada zona fueron recogidas en los mismos lugares delimitados y de la misma manera que se realizó en julio de 1994 y mayo de 1995. Las muestras corresponden a los 5 primeros centímetros del suelo. Un total de veinte muestras fueron recogidas en cada una de las zonas de estudio. Se realizaron los mismos análisis que en 1994 y 1995. En este artículo vamos a mostrar los datos referentes a estabilidad estructural y para apoyar la discusión haremos mención a otros análisis realizados en el mismo momento, como la cantidad de materia orgánica que fue determinada según el método de pérdida al fuego (Avery y Bascomb, 1974), la concentración de calcio, magnesio, hierro y aluminio a partir de ICP (Plasma de inducción), y el recubrimiento de la vegetación.

Para el análisis de esta propiedad se usaron los agregados de 4-4.8 mm. Los métodos utilizados son el TDI (diez impactos de gota) y el CND (contar el número de gotas). Los métodos simulan el impacto de gotas de lluvia. Se dejan caer gotas de $0.1 \mathrm{~g}(5.8 \mathrm{~mm}$ de diámetro) mediante una punta de bureta desde una altura de un metro sobre los agregados que están depositados en un tamiz de $2.8 \mathrm{~mm}$ de luz. El primer método consiste en saber cuánto material ha sido disgregado y ha pasado por el mismo tamiz después de que los agregados hayan sido bombardeados por diez gotas de agua cada uno de ellos. El segundo método consiste en contar el número de gotas suficiente para romper los agregados y hacerlos pasar por el tamiz. Un total de 200 agregados fueron sometidos para cada método y para cada zona. Los resultados se expresan en primer caso como porcentaje de material que ha pasado por el tamiz, y en el segundo caso como porcentaje de agregados que sobreviven a la rotura y a la disgregación (Low, 1954).

El cálculo estadístico se ha basado en la comprobación de la similitud de las medias para determinar si existen correlaciones.

$$
|\times 1-x 2| /[(\mathrm{s} 21 / \mathrm{n} 1)+(\mathrm{s} 22 / \mathrm{n} 2)] 1 / 2
$$

donde $\mathrm{x} 1 \mathrm{y}$ x 2 son las medias de los dos periodos a comparar, s1 y s2 sus correspondientes varianzas y n1 y n2 el número de valores o longitudes de cada período, respectivamente. Si el resultado de realizar la operación anterior es igual o superior a 1.96, podemos decir que las diferencias entre las medias de los dos grupos comparados son estadísticamente significativas en un $95 \%$. Si el resultado es igual o superior a 2.54 significa que las diferencias entre las medias serán estadísticamente significativas en un 99\% (Martín-Vide, 2003).

\section{Resultados y discusión}

Como se ha comentado en la metodología, el día siguiente al incendio forestal, se diseñó el muestreo y se tomaron las muestras en cada una de las cuatro zonas. Así pues los primeros análisis se realizaron sin que ningún proceso erosivo hubiese tenido lugar. Los cambios habidos en las diferentes propiedades del suelo, entre ellas la estabilidad estructural, siempre por comparación con la zona control que no sufrió el incendio, son debidos únicamente al calentamiento del suelo por el fuego.

Los resultados, que aquí llamamos 1994, son pues, estos agregados analizados justo después del fuego. Los resultados fueron los siguientes (Tabla 1): 
Tabla 1. Porcentaje de disgregación en cada una de las cuatro zonas en 1994. ** = Las diferencias son estadísticamente significativas al 99\% del nivel de confianza; no = no hay diferencias significativas.

\begin{tabular}{|c|c|c|c|c|}
\hline \multicolumn{5}{|c|}{ \% DISGREGACIÓN 1994} \\
\hline & BAJA & MEDIA & ALTA & CONTROL \\
\hline & 1.78 & 4.02 & 0.45 & 4.97 \\
\hline & 1.98 & 1.20 & 1.96 & 5.97 \\
\hline & 2.06 & 1.29 & 2.39 & 5.07 \\
\hline & 2.58 & 2.48 & 2.81 & 5.97 \\
\hline & 1.85 & 3.32 & 1.78 & 1.72 \\
\hline & 2.52 & 2.98 & 1.98 & 3.91 \\
\hline & 1.72 & 3.04 & 2.06 & 2.84 \\
\hline & 2.04 & 1.61 & 2.55 & 5.36 \\
\hline & 2.36 & 1.37 & 2.79 & 6.78 \\
\hline & 2.58 & 2.76 & 1.37 & 6.75 \\
\hline & 2.43 & 1.78 & 1.20 & 7.41 \\
\hline & 2.34 & 1.98 & 1.29 & 4.31 \\
\hline & 1.59 & 1.72 & 1,03 & 8.67 \\
\hline & 1.40 & 2.07 & 2.76 & 5.89 \\
\hline & 2.00 & 3.01 & 2.07 & 5.39 \\
\hline & 2.34 & 1.78 & 1,85 & 5.55 \\
\hline & 1.67 & 2.00 & 1.60 & 5.30 \\
\hline & 2.53 & 2.86 & 1.60 & 4.78 \\
\hline & 2.34 & 1.40 & 2.68 & 6.80 \\
\hline & 1.85 & 2.34 & 1.80 & 4.59 \\
\hline promedio & 2.10 & 2.25 & 1.90 & 5.40 \\
\hline varianza & 0.1 & 0.6 & 0.4 & 2.3 \\
\hline desv. est. & 0.4 & 0.8 & 0.6 & 1.5 \\
\hline máx. & 2.6 & 4.0 & 2.8 & 8.7 \\
\hline \multirow[t]{2}{*}{ min. } & 1.4 & 1.2 & 0.5 & 1.7 \\
\hline & BAJA & MEDIA & ALTA & \\
\hline CONTROL & $* *$ & $* *$ & $* *$ & \\
\hline BAJA & & no & no & \\
\hline MEDIA & no & & no & \\
\hline ALTA & no & no & & \\
\hline
\end{tabular}

Lo primero que debemos observar es que el porcentaje de disgregación en todas las zonas es muy bajo, con un máximo total de $8.7 \%$ en la zona control. El segundo dato es que, según este método, los agregados en 1994, justo después del fuego eran más estables, con diferencias estadísticamente significativas, que en la zona control sin quemar. Otro dato a tener en cuenta es la baja desviación entre los resultados, hay una gran homogeneidad entre todas las muestras analizadas en todas las zonas, siendo más alta también en el caso de la zona control con un valor de 1.5. 
En 1995, un año después del incendio, se volvió a muestrear y los resultados fueron los siguientes (Tabla 2):

Tabla 2. Porcentaje de disgregación en cada una de las cuatro zonas en 1995. ** = Las diferencias son estadísticamente significativas al 99\% del nivel de confianza; $*$ = Las diferencias son estadísticamente significativas al 95\% del nivel de confianza; no = no hay diferencias significativas.

\begin{tabular}{|c|c|c|c|c|}
\hline \multicolumn{5}{|c|}{ \% DISGREGACIÓN 1995} \\
\hline & BAJA & MEDIA & ALTA & CONTROL \\
\hline & 11.03 & 4.83 & 2.48 & 3.32 \\
\hline & 9.48 & 2.18 & 23.20 & 11.17 \\
\hline & 4.15 & 1.03 & 20.13 & 6.02 \\
\hline & 10.48 & 11.04 & 15.06 & 4.60 \\
\hline & 9.08 & 1.86 & 18.44 & 1.37 \\
\hline & 16.99 & 5.26 & 14.42 & 2.76 \\
\hline & 15.65 & 7.27 & 5.52 & 5.40 \\
\hline & 3.67 & 6.89 & 2.46 & 0.65 \\
\hline & 7.35 & 16.64 & 1.85 & 19.93 \\
\hline & 11.97 & 10.45 & 3.86 & 2.76 \\
\hline & 8.48 & 19.05 & 9.44 & 10.21 \\
\hline & 6.89 & 8.66 & 6.82 & 4.66 \\
\hline & 4.83 & 7.19 & 2.92 & 8.37 \\
\hline & 7.43 & 6.73 & 0.45 & 4.91 \\
\hline & 7.01 & 7.98 & 9.13 & 5.39 \\
\hline & 6.12 & 7.79 & 8.80 & 6.54 \\
\hline & 7.45 & 5.67 & 7.89 & 6.30 \\
\hline & 9.36 & 8.94 & 10.78 & 5.00 \\
\hline & 5.63 & 11.67 & 11.67 & 8.90 \\
\hline & 10.40 & 4.76 & 7.35 & 4.59 \\
\hline promedio & 8.67 & 7.79 & 9.13 & 6.14 \\
\hline varianza & 11.4 & 19.2 & 39.2 & 17.0 \\
\hline desv. est. & 3.4 & 4.4 & 6.3 & 4.1 \\
\hline máx. & 17.0 & 19.1 & 23.2 & 19.9 \\
\hline \multirow[t]{2}{*}{$\min }$. & 3.7 & 1.0 & 0.5 & 0.7 \\
\hline & BAJA & MEDIA & ALTA & \\
\hline CONTROL & $* *$ & no & $*$ & \\
\hline BAJA & & no & no & \\
\hline MEDIA & no & & no & \\
\hline ALTA & no & no & & \\
\hline
\end{tabular}


En este año se observó que las diferencias entre las tres zonas quemadas y la zona control ya no eran tan grandes como en 1994. Los máximos en las zonas habían aumentado, teniendo un máximo de $23.2 \%$ de disgregación en la zona de alta intensidad. Y también había aumentado con respecto a 1994 la desviación en todas las zonas.

En 2012 se volvieron a recoger muestras en las cuatro zonas, los resultados fueron los siguientes (Tabla 3):

Tabla 3. Porcentaje de disgregación en cada una de las cuatro zonas en 2012. ** $=$ Las diferencias son estadísticamente significativas al 99\% del nivel de confianza; $*$ = Las diferencias son estadísticamente significativas al 95\% del nivel de confianza; no = no hay diferencias significativas.

\begin{tabular}{|c|c|c|c|c|}
\hline \multicolumn{5}{|c|}{ \% DISGREGACIÓN 2012} \\
\hline & BAJA & MEDIA & ALTA & CONTROL \\
\hline & 6.21 & 16.52 & 17.45 & 3.30 \\
\hline & 11.92 & 17.41 & 9.94 & 3.73 \\
\hline & 5.01 & 10.55 & 19.63 & 2.98 \\
\hline & 14.19 & 15.40 & 7.12 & 5.04 \\
\hline & 12.92 & 19.13 & 13.98 & 5.63 \\
\hline & 7.84 & 13.76 & 7.70 & 6.26 \\
\hline & 10.22 & 11.58 & 3.80 & 3.35 \\
\hline & 22.78 & 18.96 & 10.18 & 4.23 \\
\hline & 5.42 & 10.43 & 5.00 & 4.07 \\
\hline & 8.69 & 3.71 & 19.01 & 6.29 \\
\hline & 10.10 & 6.31 & 5.32 & 3.33 \\
\hline & 5.54 & 9.85 & 13.16 & 3.71 \\
\hline & 9.68 & 10.36 & 17.81 & 1.93 \\
\hline & 3.96 & 10.57 & 11.64 & 2.61 \\
\hline & 10.81 & 14.88 & 19.47 & 4.89 \\
\hline & 2.55 & 15.66 & 9.27 & 4.83 \\
\hline & 27,56 & 11.48 & 8.81 & 6.79 \\
\hline & 23,59 & 6.28 & 11.90 & 6.73 \\
\hline & 3,67 & 10.48 & 17.18 & 1.50 \\
\hline & 13,89 & 7.96 & 20.86 & 18.18 \\
\hline promedio & 10,8 & 12.1 & 12.5 & 5.0 \\
\hline varianza & 45,4 & 17.6 & 28.1 & 11.4 \\
\hline desv. est. & 6,7 & 4.2 & 5.3 & 3.4 \\
\hline máx. & 27,6 & 19.1 & 20.9 & 18.2 \\
\hline \multirow[t]{2}{*}{$\min }$. & 2,5 & 3.7 & 3.8 & 1.5 \\
\hline & BAJA & MEDIA & ALTA & \\
\hline \multirow[t]{2}{*}{ CONTROL } & $* *$ & $* *$ & $* *$ & \\
\hline & & no & no & \\
\hline MEDIA & no & & no & \\
\hline ALTA & no & no & & \\
\hline
\end{tabular}


En 2012, dieciocho años después del incendio, el porcentaje de disgregación en las tres zonas quemadas había aumentado, con promedios superiores al 10\% en todas ellas y era significativamente mayor que en la zona control. El máximo de disgregación era superior a cualquier año de estudio, siendo de $27.6 \%$ en una muestra de la zona de baja intensidad y la desviación entre las muestras también de las más altas registradas en los tres momentos.

Para corroborar los resultados se utilizó otro método, el CND (Counting Number Drops) que también determinó que los agregados de las zonas quemadas eran bastante más inestables que los de la zona control (Fig. 3). En la primera gráfica se observa cómo los agregados que más resisten el impacto de gotas antes de romperse son los de la zona control. También los que se erosionan por completo con más dificultad son igualmente los de la zona control.
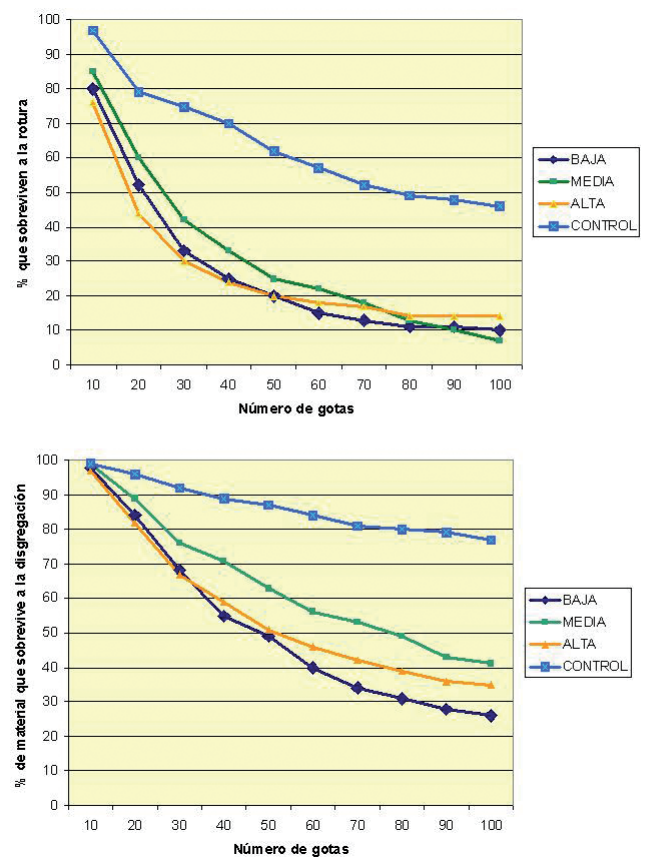

Figura 3. Resultados del método CND.

Para comprobar si las tres zonas quemadas y la zona control se habían comportado de la misma forma a lo largo del tiempo, se compararon las diferencias significativas para cada zona en todos los años (Tabla 4). Se puede comprobar que entre los tres momentos en las tres zonas quemadas ha habido variaciones más o menos significativas en la estabilidad de sus agregados respecto a la facilidad de disgregación. Estas variaciones no se observan en la zona control, cuyos datos demuestran que a lo largo del tiempo siempre han tenido el mismo grado de estabilidad. Este hecho también es importante para fortalecer la metodología usada, es decir, a pesar de que haya pasado el tiempo, los agregados del bosque no han variado su estabilidad, pues no ha habido ningún impacto para favorecer su inestabilidad. 
Tabla 4. Variaciones a lo largo del tiempo en cada una de las zonas. $* *=$ Las diferencias son estadísticamente significativas al 99\% del nivel de confianza; * = Las diferencias son estadísticamente significativas al 95\% del nivel de confianza; no = no hay diferencias significativas.

\begin{tabular}{|l|c|c|}
\hline Baja intensidad & 1995 & 2012 \\
\hline 1994 & $* *$ & $* *$ \\
\hline 1995 & & $* *$ \\
\hline Media intensidad & $* *$ & $* *$ \\
\hline 1994 & & $* *$ \\
\hline 1995 & & $*$ \\
\hline Alta intensidad & & \\
\hline 1994 & & no \\
\hline 1995 & no & no \\
\hline Bosque control & & $*$. \\
\hline 1994 & & \\
\hline 1995 & & \\
\hline
\end{tabular}

Para poder explicar qué ha ocurrido desde el incendio necesitamos más información que pasamos a exponer a continuación.

Los primeros agregados analizados (1994) son los que fueron afectados por una temperatura determinada, que en este caso fue mayor o menor dependiendo de la manera en que el incendio quemó: baja, media o alta intensidad. Como fueron suelos muestreados justo después del fuego, ninguna otra variable intervino.

Vemos, según la tabla 1, que los agregados son más estables, o sea se disgregan con mayor dificultad al impacto de gotas que los del bosque control, sin dejar de ser estos muy estables. Este resultado ha sido comprobado en muestras de incendios forestales por muchos otros investigadores, algunos hace tiempo Giovannini y Sequi (1976a, b), Ibáñez et al. (1983), Díaz-Fierros et al. (1987) y otros más recientes como el de Arcenegui et al. (2008). La explicación al porqué en algunos casos los agregados son más estables después del fuego son varias. Badia y Martí (2003) consideran que esto es así siempre que las temperaturas no son muy elevadas y no combustione por completo la materia orgánica que forma parte del agregado. Pero la más repetida es que se forma una cimentación de los óxidos existentes en el suelo, como apunta Ketterings et al. (2000), y el grado de cimentación viene determinado por la composición química del suelo y también por la cantidad de arcillas existentes. Otros autores, como Mataix-Solera y Doerr (2004), creen que la formación de una capa superficial de sustancias hidrofóbicas puede ser responsable del incremento de esta estabilidad para fuegos de baja y media intensidad.

En laboratorio también se han hecho experimentos, quemando los agregados a diferentes temperaturas. Guerrero et al. (2001), después de realizar quemas en laboratorio con suelos de bosques de Alicante, encontraron incrementos en la estabilidad de los agregados. Soto et al. (1991) hicieron varios experimentos en el laboratorio con seis suelos del 
noroeste de España y obtuvieron varios resultados: observaron un incremento de la estabilidad de los agregados en los suelos quemados hasta, como mucho, $170^{\circ} \mathrm{C}$, en cambio, en aquellos suelos quemados a temperaturas superiores, pudieron observar la destrucción de los agregados y el descenso de la estabilidad de los mismos debido a la combustión de la materia orgánica. Josa et al. (1994) encontraron un decrecimiento de la estabilidad de los agregados, quemando suelos también del noroeste de España, cuando las temperaturas alcanzaron $\operatorname{los} 200^{\circ} \mathrm{C}$ aproximadamente.

Otros estudios, en cambio, no han encontrado cambios significativos que puedan relacionarse directamente con el fuego. Es el caso de estudios como los llevados a cabo por Valzano et al. (1997) o Jordán et al. (2011) entre otros.

También hay autores que concluyen que hay un descenso de la estabilidad. Bowker et al. (2004), en un estudio con incendios forestales en Oregón, observaron un decrecimiento en la estabilidad de los agregados en los suelos quemados. Este hecho se atribuyó a la disminución de musgos y líquenes. Kavdir et al. (2005) encontraron también bajos valores de estabilidad de agregados en estudios realizados en Turquía, en zonas quemadas dos y ocho años antes del muestreo. O'Dea (2007) observó un decrecimiento en la estabilidad de los agregados dos años después de un incendio prescrito y Are et al. (2009) reportaron un descenso en esta propiedad después de un incendio forestal en Nigeria. Como puede observarse, muchos de los autores que reportan decrecimiento en la estabilidad estructural realizaron el muestreo tiempo después del incendio.

Esta gran diversidad de resultados tiene que ver con las características especiales de cada suelo, bosque, especies que habitan, tipo de incendio y otras variables como el tiempo de muestreo, procesos posteriores, prácticas post-incendio, etc. Es necesario estudiar específicamente cada caso y todos los factores que intervienen.

Por lo tanto, las causas de valores más bajos de estabilidad no sólo son atribuibles al efecto de la combustión de los agregados o al efecto de las altas temperaturas a los que fueron expuestos, sino, que participan otras variables, como el impacto de gotas de lluvia y la escorrentía superficial, que causan la erosión de la cubierta de agregados. Esta última variable, creemos que es la razón por la cual en nuestro muestreo de 1995, un año después del incendio, la estabilidad disminuyó con respecto a 1994 y también comparándolo con el bosque control. En el mismo estudio también se controló la generación de escorrentía y erosión en cada una de las cuatro zonas. Fue durante el primer año (19941995) cuando se registraron los valores más elevados. Los agregados más superficiales en las zonas quemadas tuvieron que soportar el impacto directo de las gotas de lluvia, y un lavado por una escorrentía acelerada. Algunos de ellos fueron erosionados como agregados, pues en las trampas de sedimento se podían contabilizar muchos de ellos (Tabla 5). Este hecho ya fue reportado por Bryan (1968) que encontró agregados de poco tamaño sin ningún tipo de disgregación aparente en trampas de sedimento. Hasta 1996 hubo tasas de erosión importantes, sobre todo en las zonas de media y alta intensidad (Úbeda y Sala, 1998). La tabla 5 muestra los resultados de erosión obtenidos en las dos zonas más intensamente quemadas, donde se contabilizaron los agregados mayores de 4 $\mathrm{mm}$. El hecho que haya más agregados mayores de $4 \mathrm{~mm}$ en la zona de media intensidad puede ser debido a que en la zona de alta intensidad los agregados hayan sido disgregados a tamaños más pequeños. 
Tabla 5. Peso en gramos de los agregados (>4mm) encontrados en las trampas de sedimento en las dos zonas más intensamente quemadas como consecuencia de la erosión. En la zona de baja intensidad se encontraron solamente 8.83 gramos en 1994.

\begin{tabular}{|l|c|c|}
\hline Año & Intensidad Media & Intensidad Alta \\
\hline 1994 (desde julio) & 556.51 & 303.44 \\
\hline 1995 & 96.38 & 35.21 \\
\hline 1996 & 4.56 & 1.01 \\
\hline
\end{tabular}

Este hecho tienes dos interpretaciones, la primera es que los agregados que fueron muestreados en 1994, seguramente, sobre todo en las zonas más intensamente quemadas, fueron erosionados, por tanto los agregados muestreados en 1995, probablemente en 1994 estaban unos centímetros más profundos. Creemos que la causa más importante para el decrecimiento de la estabilidad de los agregados ya no es el efecto temperatura sino el hecho de estar expuestos a las gotas de lluvia y también a la escorrentía superficial. Por tanto, cuanto más tiempo duren los procesos erosivos y la desprotección por parte de la vegetación la estabilidad de los agregados no se recuperará (Mataix-Solera y Cerdà, 2009).

No hay muchos estudios a largo plazo sobre los efectos de los incendios en el suelo. Nuestro trabajo intenta conocer cuál es la estabilidad de los agregados de un bosque que se quemó hace dieciocho años. No hay un patrón a seguir en cuanto a los cambios de la estabilidad de los agregados después de un incendio a medio y largo plazo. Algunos estudios de este tipo han dado resultados similares en estabilidad para las zonas quemadas y las zonas control (Giovannini et al., 1987) en un estudio llevado a cabo tres años después de un incendio, o Mataix-Solera et al. (2008a) quince meses después de un incendio. Otros estudios han encontrado valores más elevados en los suelos quemados como Boix Fayos (1997) o Mataix-Solera y Doerr (2004) tres años después de un incendio. Otros, en cambio, encontraron valores más bajos, Bowker et al. (2004) once meses después del fuego, Kavdir et al. (2005) doce años después del incendio o Mataix-Solera et al. (2008b) diecinueve años después de un incendio. Granged et al. (2011) observaron un decrecimiento de la estabilidad de los agregados después del incendio, en cambio, tres años después, los valores habían aumentado ligeramente. Este aumento puede haber sido ocasionado por la erosión de los agregados más inestables de la capa más superficial del suelo, dejando agregados más superficiales que nunca fueron afectados por la temperatura.

En nuestro caso, los resultados obtenidos en 2012, dieciocho años después del fuego, muestran grandes diferencias entre la zona control y las zonas quemadas. Se observa que los agregados de la zona control son más resistentes. En cambio, los agregados de las zonas quemadas en media y alta intensidad son, de media, muy similares y son los más débiles de las tres zonas quemadas.

Para explicar el porqué dieciocho años después del incendio los agregados muestran estabilidades menores que en 1995 y que en la zona control, otras propiedades se deben tener en cuenta. Varios autores como Sanroque et al. (1985), Giovannini et al. (1987), Cerdà (1993) o Badia y Martí (2003) han observado disminuciones en la estabilidad de agregados relacionadas con la pérdida de materia orgánica. Nadal-Romero et al., (2009) encontraron que el contenido de materia orgánica y de carbonatos muestra también un efecto positivo sobre la estabilidad de los agregados. En nuestro caso es cierto que los agregados de la zona quemada contenían un menor porcentaje de materia orgánica. En la 
zona control se obtuvo un promedio de $19.18 \%$ de materia orgánica, mientras que en la zona de baja intensidad era de $12.87 \%$, en la media, $9.22 \%$ y en la zona de alta intensidad de quemado de $11.11 \%$.

Como en la literatura sobre el tema muchos autores afirman que los óxidos metálicos pueden ser muy importantes para la agregación y consistencia de los agregados (Singer, 1994, Ketterings et al., 2000; Mataix-Solera et al., 2011; Campo et al., 2014), se analizó el aluminio y el hierro de las muestras y como se puede comprobar la cantidad de, sobre todo, hierro, es muy superior en la zona control, lo que explicaría otra razón de por qué los agregados del bosque sin quemar son más estables (Tabla 6).

Tabla 6. Concentración en $\mathrm{mg} \mathrm{kg}^{-1}$ de aluminio y hierro en las muestras de suelo de cada una de las zonas de estudio.

\begin{tabular}{|l|c|c|c|c|}
\hline & Baja & Media & Alta & Control \\
\hline Aluminio & 3.61 & 2.54 & 1.55 & 3.84 \\
\hline Hierro & 3.72 & 3.28 & 1.36 & 11.08 \\
\hline
\end{tabular}

No sólo la cantidad de estos elementos es importante. Barthès et al. (2008) indican que gran parte del protagonismo que se le ha dado a la materia orgánica como aglutinador de partículas y consecuentemente de buen agente agregador, sin elementos como el aluminio y hierro, pierde capacidad de cimentación. No es el único autor que así lo determina. Igwe et al. (2009), en estudios realizados en Nigeria, también concluyen que el papel del carbón orgánico no es determinante si no hay elementos que pueden llegar a oxidarse.

Estos autores también remarcan la importancia de los cationes básicos, sobre todo calcio y magnesio para la agregación de las partículas. En nuestro caso, todos los elementos son mayoritarios en la zona control, lo que puede ser determinante para que los agregados sean más resistentes al impacto de las gotas en esta zona.

Otra hipótesis es que el resurgimiento de mucha vegetación herbácea y arbustiva en las zonas quemadas haya tenido un efecto sobre la agregación, desestructurando los primeros centímetros del suelo y afectando a la estabilidad de los agregados. Fattet et al. (2011) han encontrado que en suelos de China, así como hay vegetación que puede proteger el suelo a la desestructuración, hay otro tipo de vegetación que en su estado de resurgimiento pueden causar incluso un efecto contrario a la agregación. Esta vegetación suelen ser árboles, arbustos o herbáceas de crecimiento rápido.

Cerdà (1998) ya había determinado que los agregados desarrollados en vegetación como Rosmarinus officinalis, Thymus vulgaris, Ulex parviflorus y Anthyllis cystisoides eran menos resistentes que los agregados en bosques más maduros de Quercus ilex. Esta vegetación, que aparece después de incendios forestales, está muy presente en las tres áreas quemadas, lo que explicaría este porcentaje menor de estabilidad en los agregados que en el suelo del bosque control.

Creemos que el hecho de que haya más elementos químicos (calcio, magnesio, hierro y aluminio) en el suelo control que en las zonas quemadas, así como también más 
materia orgánica, hace que los agregados de la zona control sean más resistentes. Después de dieciocho años, el suelo quemado no ha alcanzado valores de concentración pre-quema. Seguramente nunca esta zona será como la zona control, pues ésta es una plantación de pinos, y el bosque que está recuperándose en la zona quemada será un bosque mixto de alcornoques y pinos con un gran sotobosque de arbustos y herbáceas.

El hecho de que el recubrimiento por parte de la vegetación sea del 100\%, hace que no haya un peligro de disgregación de estos agregados, pues están muy protegidos frente a la acción de la lluvia.

\section{Conclusiones}

Dieciocho años después del incendio la estabilidad de los agregados del suelo quemado es diferente a la de la zona control que no sufrió el incendio. Varias razones son las que hacen más estables los agregados superficiales de la zona control, entre ellas que el suelo superficial de esta zona tiene más materia orgánica, más calcio y magnesio, que son elementos que favorecen la agregación y ha sido menos agredida, por el fuego primero, por la erosión después y por un proceso de revegetación después.

Las diferencias entre cada una de las tres zonas quemadas después de dieciocho años no son estadísticamente significativas, y parece que hay una cierta homogeneización entre las tres (baja, media y alta intensidad de fuego).

A pesar de que los agregados más superficiales de esta zona quemada son menos resistentes al impacto de gota, no creemos que se produzca en la actualidad una gran disgregación cuando hay precipitaciones, pues la superficie del suelo de las zonas quemadas está muy protegida por la abundante vegetación aparecida después del fuego. Lo que sí existe en el actual estado de este bosque es el riesgo de que en caso de incendio forestal, éste alcance dimensiones de Gran Incendio Forestal (GIF) pues la cantidad de combustible es muy abundante.

\section{Agradecimientos}

Al "Comissionat per a Universitats i Recerca del DIUE de la Generalitat de Catalunya" por subvencionar durante el periodo 2009-2013 al GRAM (Grup de Recerca Ambiental Mediterrània) SGR2009:1515. Queríamos agradecer también las aportaciones que han realizado los dos revisores anónimos que sin duda han ayudado a que este artículo fuese de mayor calidad.

\section{Referencias bibliográficas}

Arcenegui, V., Mataix-Solera, J., Guerrero, C., Zornoza, R., Mataix-Beneyto, J., García-Orenes, F. 2008. Immediate effects of wildfires on water repellency and aggregate stability in Mediterranean calcareous soils. Catena 74, 219-226.

Are, K.S., Oluwatosin, G.A., Adeyolanu, O.D., Oke, A.O. 2009. Slash and burn effects on soil quality of an Alfisol: soil physical properties. Soil and Tillage Research 103, 4-10. 
Avery, B.W., Bascomb, C.L. 1974. Soil survey laboratory methods. Soil Survey Tech. Monogr. No. 6. Rothamsted Exp. Harpenden, UK, pp. 19-25.

Badía, D., Martí, C. 2003. Plant ash and heat intensity effects on chemical and physical properties of two contrasting soils. Arid Land Research and Management 17, 23-41.

Barthès, B.G., Kouakoua, E., Larré-Larrouy, M.C., Razafimbelo, T.M., de Luca, E.F., Azontonde, A., Neves, C., Freitas, P.L., Feller, C.L. 2008. Texture and sesquioxide effects on water-stable aggregates and organic matter in some tropical soils. Geoderma 143, 14-25.

Benito, E, Gómez, A., Díaz-Fierros, F. 1986. Descripción de un simulador de lluvia para estudios de erodibilidad del suelo y estabilidad de los agregados al agua. Anales de Edafología y Agrobiología 9-10, 1115-1126.

Boix Fayos, C. 1997. The roles of texture and structure in the water retention capacity of burnt Mediterranean soils with varying rainfall. Catena 31, 219-236.

Bowker, M.A., Belnap, J., Rosentreter, R., Graham, B. 2004. Wildfire-resistant biological soil crusts and fire-induced loss of stability in Palouse prairies, USA. Applied Soil Ecology 26, 41-52.

Bryan, R.B. 1968. The development use and eficiency of indices of soil erodibility. Geoderma 2, 5-26.

Bryan, R.B. 2000. Soil erodibility and processes of water erosion on hill slope. Geomorphology 32, 385-415.

Campo, J., Gimeno-García, E., Andreu, V., González-Pelayo, O., Rubio, J.L. 2014. Cementing agents involved in the macro- and microaggregation of a Mediterranean shrubland soil under laboratory heating. Catena 113, 165-176.

Cerdà, A. 1993. Incendios forestales y estabilidad de los agregados. Cuadernos de Geografía 53, 1-16.

Cerdà, A. 1998. Soil aggregate stability under different Mediterranean vegetation types. Catena 32, 73-86.

Cerdà, A., Jordán, A. 2010. Actualización en métodos y técnicas para el estudio de los suelos afectados por incendios forestales. Cátedra de Divulgació de la Ciència, Universitat de València, $521 \mathrm{pp}$.

Certini, G. 2005. Effects of fire on properties of forest soils: a review. Oecologia 143, 1-10.

Díaz-Fierros, F., Benito Rueda, E., Pérez Moreira, R. 1987. Evaluation of the U.S.L.E. for the prediction of erosion in burnt forest areas in Galicia (N.W. Spain). Catena 14, 189-199.

Edwards, A.P., Bremner, J.M. 1967. Dispersion of soil particles by sonic vibration. Journal of Soil Science 18, 47-63.

Fattet, M., Ghestem, Y.F.M., Ma, W., Foulonneau, M., Nespoulous, J., Bissonnais, Y Le., Stokes, A. 2011. Effects of vegetation type on soil resistance to erosion: Relationship between aggregate stability and shear strength. Catena 87 (1), 60-69.

Giovannini, G., Sequi, P. 1976a. Iron and aluminium as cementing substances of soil aggregates. I. Acetylacetone in benzene as an extractant of fractions of soil iron and aluminium. Journal of Soil Science 27, 140-147.

Giovannini, G., Sequi, P. 1976b. Iron and aluminium as cementing substances of soil aggregates. II. Changes in stability of soil aggregates following extraction of iron and aluminium by acetylacetone in a no polar solvent. Journal of Soil Science 27, 148-153.

Giovannini, G., Lucchesi, S. 1997. Modifications induced in soil physico-chemical parameters by experimental fires at different intensities. Soil Science 162, 479-486.

Giovannini, G., Lucchesi, S., Giachetti, M. 1987. The natural evolution of a burnt soil: a three-year investigation. Soil Science 143, 220-226. 
Granged, A.J.P., Zavala, L.M., Jordán, A., Bárcenas-Moreno, G. 2011. Post-fire evolution of soil properties and vegetation cover in a Mediterranean heathland after experimental burning: A 3-year study. Geoderma 164, 85-94.

Guerrero, C., Mataix-Solera, J., García-Orenes, F., Gómez, I., Navarro-Pedreño, J. 2001. Different patterns of aggregate stability in burned and restored soils. Arid Land Research and Management 15, 163-171.

Harris, R.F., Chesters, G., Allen, O.N., Attoe, O.J. 1964. Mechanisms involved in soil aggregate stabilization by fungi and bacteria. Soil Science Society of America Proceedings 28, 529-532.

Hillel, D. 1998. Environmental Soil Physics. Academic Press, San Diego, 771 pp.

Holz, S.C., Ingelmo, F., Canet, R. 2000. Long term effects of the application of sewage sludge and vegetal cover on some physical and physicochemical properties of a degraded arid soil. Agrochimica 44, 132-139.

Hoogmoed, W.B., Stroosnijder, L. 1984. Crust formation on sandy soils in the Sahel. Soil \& Tillage Research 4, 5-24.

Ibáñez, J.J., Lobo, M.C., Almendros, G.., Polo, A. 1983. Impacto del fuego sobre algunos ecosistemas edáficos de clima mediterráneo continental en la zona centro de España. Boletín de la Estación central de Ecología 24, 755-777.

Igwe, C.A., Zarei, M., Stahr, K. 2009. Colloidal stability in some tropical soils of southeastern Nigeria as affected by iron and aluminium oxides. Catena 77 (3), 232-237.

Imeson, A.C. 1984. An eco-geomorphological approach to the soil degradation and erosion problem. En Desertification in Europe, R. Fantechi, N.S. Margaris (eds.), D. Reidel Publishing Co. Dordrecht, pp. 110-125.

Imeson, A.C., Vis, M. 1984. Assessing soil aggregate stability by water-drop impact and ultrasonic dispersion. Geoderma 34, 185-200.

Insam, H., Domsch, K.H. 1988. Relationship between soil organic carbon and microbial biomass on chronosequences of reclamation sites. Microbial Ecology 15, 177-188.

Jordán, A., Zavala, L.M., Mataix-Solera, J., Nava, A.L., Alanís, N. 2011. Effect of fire severity on water repellency and aggregate stability on Mexican volcanic soils. Catena 84, 136-147.

Josa, R., Arias, X., Solé, A. 1994. Effects of slashburning on some osil physical properties in an olm-oak coppice. En Soil erosion and degradation as a consequence of forest fires, M. Sala., J. L. Rubio (eds.), Geoforma Ediciones, Logroño, pp. 29-42.

Kavdir, Y., Ekinci, H., Yüksel, O., Mermut, A.R. 2005. Soil aggregate stability and ${ }^{13} \mathrm{C}$ CP/MASNMR assessment of organic matter in soils influenced by forest wildfires in Çanakkale, Turkey. Geoderma 129, 219-229.

Kemper, M.D., Koch, E.J. 1966. Aggregate stability of soils from Western United States and Canada. United States Department of Agriculture. Technical Bulletin $\mathrm{N}^{\circ} 1355$.

Kemper, M.D., Rosenau, R.C. 1986. Aggregate stability and size distribution. A: Methods of Soils Analysis. Part I. Physical and Mineralogical Methods. Agronomy Monograph 9.

Ketterings, Q.M., Bigham, J.M., Laperche, V. 2000. Changes in soil mineralogy and texture caused by slash-and-burn fires in Sumatra, Indonesia. Soil Science Society of America Journal 64, 1108-1117.

Low, A.J. 1954. Study of soil structure in field and laboratory. Journal of Soil Science 5, 19-54.

Martín-Vide, J.M. 2003. El tiempo y el clima. Barcelona. Rubes, Barcelona, 127 pp.

Mataix-Solera, J., Doerr, S.H. 2004. Hydrophobicity and aggregate stability in calcareous topsoils from fire-affected pine forests in southeastern Spain. Geoderma 118, 77-88.

Mataix-Solera, J., Guerrero, C. 2007. Efectos de los incendios forestales en las propiedades edáficas. En Incendios Forestales, Suelos y Erosión Hídrica, J. Mataix-Solera (ed.), Caja Mediterráneo CEMACAM, Alicante, pp. 5-40. 
Mataix-Solera, J., Cerdà, A. 2009. Incendios forestales en España. Ecosistemas terrestres y suelos. En Efectos de los incendios forestales sobre los suelos en España, A. Cerdà, J. Mataix-Solera (eds.). Cátedra Divulgación de la Ciencia. Universitat de València, pp. 13-53.

Mataix-Solera, J., Navarro, M.A., Zornoza, R., Guerrero, C., Gómez, I., García-Orenes, F., Bárcenas, G. M. 2008a. Fire effects on soil organic carbon in turn affecting physical, chemical and biochemical soil properties. Geophysical Research Abstracts 10, 01280.

Mataix-Solera, J., Arcenegui, V., Guerrero, C., Jordan, M.M., Dalpa, P., Tessler, N., Wittenberg, L. 2008b. Can terra rosa become water repellent by burning? A laboratory approach. Geoderma 157, 178-184.

Mataix-Solera, J., Benito, E., Andreu, B., Cerdà, A., Llovet, J., Úbeda, X., Martí, C., Varela, E., Gimeno, E., Arcenegui, V., Rubio, J.L., Campo, J., García-Orenes, F., Badía, D. 2010. ¿Cómo estudiar la estabilidad de agregados en suelos afectados por incendios? Métodos e interpretación de resultados. En Actualización en métodos y técnicas para el estudio de los suelos afectados por incendios forestales, A. Cerdà, A. Jordán (eds.), Càtedra de Divulgació de la Ciència, Universitat de València, pp. 111-143.

Mataix-Solera, J., Cerdà, A., Arcenegui, V., Jordán, A., Zavala, L.M. 2011. Fire effects on soil aggregation: A review. Earth-Science Reviews 109, 44-60.

McIntyre, D.S. 1958. Permeability measurements of soil crust formed by raindrop impact. Soil Science 85, 185-189.

Moreno, J.M., Oechel, W.C. 1989. A simple method for estimating fire intensity after a burnt in California Chaparral. Acta Oecologica 10 (1), 57-68.

Nadal-Romero, E., Regüés, D., Salvador Sanchís, P., Torri, D. 2009. La estabilidad de los agregados del suelo en ambientes forestales y acarcavados del Pirineo Central. Cuaternario y Geomorfología 23 (1-2), 49-64.

Oades, J.M. 1993. The role of biology in the formation, stabilization and degradation of soil structure. Geoderma 56, 377-400.

O’Dea, M.E. 2007. Fungal mitigation of soil erosion following burning in a semi-arid Arizona savanna. Geoderma 138, 79-85.

Sanchís, M.P., Torri, D., Borselli, L., Poesen, J. 2008. Climate effects on soil erodibility. Earth Surface Processes and Landforms 33 (7), 1082-1097.

Sanroque, P., Rubio, J. L., Mansanet, J. 1985. Changes in soil properties, floristic composition and water erosion following mediterranean forest fires in Valencia (Spain). Revue d'Ecologie et Biologie du Sol 22, 131-147.

Singer, A. 1994. Clay mineralogy as affecting dispersivity and crust formation in Aridisols. En Transactions of the 15th World Congress of Soil Science, J. D. Etchevers (ed.), Acapulco, Mexico, pp. 37-46.

Soto, B., Benito, E., Diaz-Fierros, F. 1991. Heat-induced degradation processes in forst soils. International Journal of Wildland Fire 1, 147-152.

Úbeda, X. 1998. Efectes de les diferents intensitats de foc, durant els incendis forestals, en els paràmetres físics $i$ químics del sòl i en l'increment de l'escolament i de l'erosió. Tesis Doctoral. Universitat de Barcelona, $231 \mathrm{pp}$.

Úbeda, X., Sala, M. 1998. Variations in runoff and erosion in three areas with different fire intensity. Geoökodynamik 19, 179-188.

Valzano, F.P., Greense, R.S.B., Murphy, B.W. 1997. Direct effects of stubble burning on soil hydraulic and physical properties in a direct drill tillage system. Soil and Tillage Research 42, 209-219.

Yoder, R.E. 1936. A direct method of aggregate analysis of soils and a study of the physical nature of erosion losses. Journal of the American Society of Agronomy 28, 337-351. 